\title{
Upper bound for the Gromov width of flag manifolds
}

\author{
Alexander Caviedes Castro
}

\begin{abstract}
We find an upper bound for the Gromov width of coadjoint orbits of $U(n)$ with respect to the Kirillov-Kostant-Souriau symplectic form by computing certain Gromov-Witten invariants. The approach presented here is closely related to the one used by Gromov in his celebrated non-squeezing theorem.
\end{abstract}

\section{Introduction}

The Darboux theorem in symplectic geometry states that around any point of a symplectic manifold there is a system of local coordinates such that the symplectic manifold looks locally like $\mathbb{R}^{2 n}$ with its canonical symplectic form. A natural and fundamental problem in symplectic geometry is to know how far we can extend symplectically these coordinates in the symplectic manifold. This is how the concept of Gromov's width arises. The Gromov width of a symplectic manifold $\left(M^{2 n}, \omega\right)$ is defined as

$$
\operatorname{Gwidth}\left(M^{2 n}, \omega\right):=\sup \left\{\pi r^{2}: \exists \text { a symplectic embedding } B^{2 n}(r) \hookrightarrow M^{2 n}\right\},
$$

where $B^{2 n}(r)$ denotes the open ball of radius $r$ and center the origin in $\mathbb{R}^{2 n}$ endowed with the standard symplectic form.

The Gromov non-squeezing theorem gives us insights of how restrictive is the Gromov width from above. It says that if there is a symplectic embedding of the ball $B^{2 n}(r) \subset \mathbb{R}^{2 n}$ of radius $r$ into a cylinder $B^{2}(\lambda) \times \mathbb{R}^{2 n-2}$ of radius $\lambda$ with respect to the standard symplectic forms, then $r \leq \lambda$. In particular,

$$
\operatorname{Gwidth}\left(B^{2}(\lambda) \times \mathbb{R}^{2 n-2}\right)=\pi \lambda^{2} .
$$

Gromov's non-squeezing theorem was proven in [5], where the connection between $J$-holomorphic curves and sympletic geometry is established. Since then, several authors have used Gromov's method for bounding the Gromov width of other families of symplectic manifolds, such as G. Lu for symplectic toric manifolds in [14]; G. Lu, and Karshon and Tolman for 
complex Grassmannians manifolds in [13], and in [10], respectively; and Zoghi for regular coadjoint orbits in [22] (see also McDuff and Polterovich [15], Biran [2]).

In this paper, we want to find upper bounds for the Gromov width of unitary orbits on Hermitian matrices. For $\lambda=\left(\lambda_{1}, \ldots, \lambda_{n}\right) \in \mathbb{R}^{n}$, let

$$
\mathcal{H}_{\lambda}:=\left\{A \in M_{n}(\mathbb{C}): A^{*}=A, \text { spectrum } A=\lambda\right\} .
$$

The set of Hermitian matrices $\mathcal{H}_{\lambda}$ can be identified with a coadjoint orbit of $U(n)$ and we can endow $\mathcal{H}_{\lambda}$ with a symplectic form $\omega_{\lambda}$ coming from the Kostant-Kirillov-Souriau symplectic form defined on the coadjoint orbit. The next is the main result obtained in this paper:

Main Theorem. Let $\lambda=\left(\lambda_{1}, \ldots, \lambda_{n}\right) \in \mathbb{R}^{n}$. Suppose that there are integers $1 \leq i, j \leq n$ such that any difference of eigenvalues $\lambda_{i^{\prime}}-\lambda_{j^{\prime}}$ is an integer multiple of $\lambda_{i}-\lambda_{j}$, then

$$
\operatorname{Gwidth}\left(\mathcal{H}_{\lambda}, \omega_{\lambda}\right) \leq\left|\lambda_{i}-\lambda_{j}\right| .
$$

In his Ph.D thesis [22], Zoghi has shown the inequality appearing in the Main Theorem when all components of $\lambda \in \mathbb{R}^{n}$ are pairwise different. In this paper, we extend Zoghi's theorem when $\lambda \in \mathbb{R}^{n}$ is not necessarily of this form.

This paper is organized as follows: we first introduce the $J$-holomorphic tools that we will use throughout the text and explain how they are related with the Gromov width of a symplectic manifold. Then we show how to bound from above the Gromov width of a Grassmannian manifold by computing certain Gromov-Witten invariant. Finally, we explain how to determine upper bounds for the Gromov width of flag manifolds by computing GromovWitten invariants on holomorphic fibrations whose fibers are isomorphic to Grassmannian manifolds.

We suggest to the reader to compare our results with the ones obtained by Pabiniak in [17] and [18], where she has considered the problem of determining lower bounds for the Gromov width of flag manifolds. In her papers, Pabiniak has proved that for any $\lambda=\left(\lambda_{1}, \ldots, \lambda_{n}\right) \in \mathbb{R}^{n}$,

$$
\operatorname{Gwidth}\left(\mathcal{H}_{\lambda}, \omega_{\lambda}\right) \geq \min _{\lambda_{i} \neq \lambda_{j}}\left|\lambda_{i}-\lambda_{j}\right| .
$$

This result together with the Main Theorem implies that the upper bound that we found is indeed the Gromov width of $\left(\mathcal{H}_{\lambda}, \omega_{\lambda}\right)$. 


\section{2. $J$-holomorphic curves}

In this section we give a short review of pseudoholomorphic theory and Gromov-Witten invariants, and we show how they are related with the Gromov width of a symplectic manifold. Most of the material presented here is adapted from McDuff and Salamon [16].

Let $\left(M^{2 n}, \omega\right)$ be a symplectic manifold. An almost complex structure $J$ of $(M, \omega)$ is a smooth operator $J: T M \rightarrow T M$ such that $J^{2}=-I d$. We say that an almost complex structure $J$ is compatible with $\omega$ if the formula

$$
g(v, w):=\omega(v, J w)
$$

defines a Riemannian metric. We denote the space of $\omega$-compatible almost complex structures by $\mathcal{J}(M, \omega)$.

Let $\left(\mathbb{C P}^{1}, j\right)$ be the Riemann sphere with its standard complex structure $j$. Let $J \in \mathcal{J}(M, \omega)$. A map $u: \mathbb{C P}^{1} \rightarrow M$ is called a $J$-holomorphic curve of genus zero or simply a $J$-holomorphic curve if

$$
J \circ d u=d u \circ j .
$$

A curve $u: \mathbb{C P}^{1} \rightarrow M$ is said to be multiply covered if it is the composite of a holomorphic branched covering map $\left(\mathbb{C P}^{1}, j\right) \rightarrow\left(\mathbb{C P}^{1}, j\right)$ of degree greater than one with a $J$-holomorphic map $\mathbb{C P}^{1} \rightarrow M$. It is simple if it is not multiply covered.

Given a compact symplectic manifold $\left(M^{2 n}, \omega\right)$, a compatible almost complex structure $J \in \mathcal{J}(M, \omega)$, and a second homology class $A \in H_{2}(M, \mathbb{Z})$, we define the moduli space of simple $J$-holomorphic curves of degree $A$ as

$$
\mathcal{M}_{A}^{*}(M, J):=\left\{u: \mathbb{C P}^{1} \rightarrow M: J \circ d u=d u \circ j, u_{*}\left[\mathbb{C P}^{1}\right]=A, u \text { is simple }\right\}
$$

The moduli space of simple $J$-holomorphic curves of degree $A$ with $k$-marked points is defined by

$$
\mathcal{M}_{A, k}^{*}(M, J):=\mathcal{M}_{A}^{*}(M, J) \times_{P S L(2, \mathbb{C})}\left(\mathbb{C P}^{1}\right)^{k}
$$

where $P S L(2, \mathbb{C})$ acts on the right factor by its natural action on $\mathbb{C P}^{1}$ and on the left factor by reparametrization. When $k=0$, we define $\mathcal{M}_{A, 0}^{*}(M, J)$ 
as being equal to $\mathcal{M}_{A}(M, J) / P S L(2, \mathbb{C})$. We also have an evaluation map

$$
\begin{aligned}
\operatorname{ev}_{J}^{k}: \mathcal{M}_{A, k}^{*}(M, J) & \rightarrow M^{k} \\
{\left[u,\left(z_{1}, \ldots, z_{k}\right)\right] } & \mapsto\left(u\left(z_{1}\right), \ldots, u\left(z_{k}\right)\right) .
\end{aligned}
$$

Let $\mathcal{J}_{\text {reg }}(M, \omega) \subset \mathcal{J}(M, \omega)$ denote the set of regular almost complex structures (see e.g. McDuff and Salamon [16, Definition 3.1.4]). The set $\mathcal{J}_{\text {reg }}(M, \omega)$ is residual in the set $\mathcal{J}(M, \omega)$ of compatible almost complex structures, i.e., it contains a countable intersection of open dense sets with respect to the $C^{\infty}$ topology. For $J \in \mathcal{J}_{\text {reg }}(M, \omega)$, the moduli space $\mathcal{M}_{A, k}^{*}(M, J)$ is a smooth oriented manifold of dimension equal to $\operatorname{dim} M+2 c_{1}(A)+2 k-$ 6 , where $c_{1}$ denotes the first Chern class of the bundle $(T M, J)$ (see e.g. McDuff and Salamon[16, Theorem 3.1.5]).

Remark 2.1. If $(M, \omega, J)$ is a compact Kähler manifold and $G$ is a Lie group that acts transitively on $M$ by $J$-holomorphic diffeomorphism, then the almost complex structure $J$ is regular (McDuff and Salamon [16, Proposition 7.4.3]).

The Gromov width of a symplectic manifold $\left(M^{2 n}, \omega\right)$ is defined as $\operatorname{Gwidth}\left(M^{2 n}, \omega\right)=\sup \left\{\pi r^{2}: \exists\right.$ a symplectic embedding $\left.B^{2 n}(r) \hookrightarrow M^{2 n}\right\}$, where $B^{2 n}(r)$ denotes the open ball of radius $r$ and center the origin in $\mathbb{R}^{2 n}$ endowed with the standard symplectic form.

The Darboux theorem implies that the Gromov width of a symplectic manifold is always positive. Moreover, if the symplectic manifold is compact, its Gromov's width is finite.

The following statement shows the relation between pseudoholomorphic curves and Gromov's widths:

Theorem 2.2. Let $\left(M^{2 n}, \omega\right)$ be a compact symplectic manifold, and $A \in$ $H_{2}(M, \mathbb{Z}) \backslash\{0\}$ a second homology class. Suppose that for a dense subset of smooth $\omega$-compatible almost complex structures, the evaluation map

$$
\operatorname{ev}_{J}^{1}: \mathcal{M}_{A}^{*}(M, J) \times_{P S L(2, \mathbb{C})} \mathbb{C P}^{1} \rightarrow M
$$

is onto. Then for any symplectic embedding $B^{2 n}(r) \hookrightarrow M$, we have

$$
\pi r^{2} \leq \omega(A)
$$


where $\omega(A)$ denotes the symplectic area of $A$. In particular,

$$
\operatorname{Gwidth}(M, \omega) \leq \omega(A)
$$

Proof. See e.g. Zoghi [22, Proposition 3.6].

A homology class $B \in H_{2}(M)$ is spherical if it is in the image of the Hurewicz homomorphism $\pi_{2}(M) \rightarrow H_{2}(M)$. A homology class $B \in H_{2}(M)$ is $\omega$-indecomposable if it does not decompose as a sum $B=B_{1}+\cdots+B_{k}$ of spherical classes such that $\omega\left(B_{i}\right)>0$.

A symplectic manifold $\left(M^{2 n}, \omega\right)$ is semipositive if, for a spherical homology class $A$ with positive symplectic area, $c_{1}(A) \geq 3-n$ implies $c_{1}(A)$ $\geq 0$.

If we assume that either the symplectic manifold $(M, \omega)$ is semipositive or the homology class $A \in H_{2}(M, \mathbb{Z})$ is $\omega$-indecomposable, for a regular almost complex structure $J \in \mathcal{J}_{\text {reg }}(M, \omega)$, the evaluation map

$$
\mathrm{ev}_{J}^{k}: \mathcal{M}_{A, k}^{*}(M, J) \rightarrow M^{k}
$$

represents a pseudocycle, i.e., its image can be compactified by adding a set of codimension at least two. This pseudocycle can be used to define the Gromov-Witten invariants: If $a_{i} \in H^{*}(M, \mathbb{Z})$ are cohomology classes Poincaré dual to compact oriented submanifolds $X_{i} \subset M$, the GromovWitten invariant $\mathrm{GW}_{A, k}^{J}\left(a_{1}, \ldots, a_{k}\right)$ is the number of $J$-holomorphic curves in the class $A$ passing through the submanifolds $X_{i}$ (after possibly perturbing them) and counted with appropriate signs. More precisely, if $\sum_{i=1}^{k} \operatorname{deg} a_{i}=$ $\operatorname{dim} \mathcal{M}_{A, k}^{*}(M, J)$ and if the moduli space $\mathcal{M}_{A, k}^{*}(M, J)$ is endowed with a suitable orientation (see e.g. McDuff and Salamon [16, Section A.2]); the Gromov-Witten invariant is defined as the oriented intersection number

$$
\mathrm{GW}_{A, k}^{J}\left(a_{1}, \ldots, a_{k}\right):=\sharp \operatorname{ev}_{J}^{k} \pitchfork\left(X_{1} \times \cdots \times X_{k}\right) .
$$

Gromov-Witten invariants $\mathrm{GW}_{A, k}^{J}$ are well-defined, finite and independent of the regular almost complex structure $J$ when we assume that either the symplectic manifold $(M, \omega)$ is semipositive or $A$ is $\omega$-indecomposable (McDuff and Salamon [16, Theorem 7.1.1, Lemma 7.1.8]). 
Remark 2.3. If there exist cohomology classes $a_{1}, \ldots, a_{k} \in H^{*}(M, \mathbb{R})$ and a regular almost complex structure $J$ such that

$$
\mathrm{GW}_{A, k}^{J}\left(a_{1}, \ldots, a_{k}\right) \neq 0
$$

and $a_{1}$ is Poincaré dual to the fundamental class of a point, then for generic almost complex structure $J^{\prime} \in \mathcal{J}(M, \omega)$, the evaluation map

$$
\operatorname{ev}_{J^{\prime}}^{1}: \mathcal{M}_{A, 1}^{*}\left(M, J^{\prime}\right) \rightarrow M
$$

is onto, which, by Theorem 2.2, implies that

$$
\operatorname{Gwidth}(M, \omega) \leq \omega(A) .
$$

Remark 2.4. Gromov-Witten invariants for symplectic manifolds can be defined in wide generality by associating to the moduli spaces of $J$ holomorphic curves virtual fundamental classes with rational coefficients ( $\mathrm{Li}$ and Tian [12], Fukaya and Ono [4, Ruan [19], Siebert [20, Hofer,Wysocki and Zehnder [8], [9]). We will make no use of this definition since we want to keep as simple and self-contained as possible the presentation of this paper. However, with this definition we would not need to assume that either the symplectic manifold is semipositive or the homology class $A$ is $\omega$-indecomposable, and the results of Theorem 5.3 can be extended to any coadjoint orbit of type A.

\section{Flag manifolds}

In this section we recall some general statements about coadjoint orbits and unitary orbits on Hermitian matrices. Most of the material shown here can be found in the classical literature such as Kirillov [11].

Let $G$ be a compact Lie group, $\mathfrak{g}$ be its Lie algebra, and $\mathfrak{g}^{*}$ be the dual of the Lie algebra $\mathfrak{g}$. The compact Lie group $G$ acts on $\mathfrak{g}^{*}$ by the coadjoint action. Let $\xi \in \mathfrak{g}^{*}$ and $\mathcal{O}_{\xi}$ be the coadjoint orbit passing through $\xi$.

The coadjoint orbit $\mathcal{O}_{\xi}$ carries a symplectic form defined as follows: for $\xi \in \mathfrak{g}^{*}$ we define a skew bilinear form on $\mathfrak{g}$ by

$$
\omega_{\xi}^{K K S}(X, Y)=\langle\xi,[X, Y]\rangle .
$$

The kernel of $\omega_{\xi}^{K K S}$ is the Lie algebra $\mathfrak{g}_{\xi}$ of the stabilizer of $\xi \in \mathfrak{g}^{*}$ for the coadjoint representation. In particular, $\omega_{\xi}^{K K S}$ defines a nondegenerate skewsymmetric bilinear form on $\mathfrak{g} / \mathfrak{g}_{\xi}$, a vector space that can be identified with 
$T_{\xi}\left(\mathcal{O}_{\xi}\right) \subset \mathfrak{g}^{*}$. The bilinear form $\omega_{\xi}^{K K S}$ induces a closed, invariant, nondegenerate 2 -form on the orbit $\mathcal{O}_{\xi}$, therefore defining a symplectic structure on $\mathcal{O}_{\xi}$. This symplectic form is known as the Kostant-Kirillov-Souriau form of the coadjoint orbit.

Let us assume now that $G=U(n)$. Let $\mathfrak{u}(n)$ be the Lie algebra of $U(n)$, $\mathfrak{u}(n)^{*}$ be its dual and $\mathcal{H}:=\left\{A \in M_{n}(\mathbb{C}): A^{*}=A\right\}$ be the set of Hermitian matrices.

The group of unitary matrices $U(n)$ acts by conjugation on $\mathcal{H}$. The Hermitian matrices $\mathcal{H}$ have real eigenvalues and are diagonalizable in a unitary basis, so that the orbits of this action correspond to sets of matrices in $\mathcal{H}$ with the same spectrum. Let $\lambda=\left(\lambda_{1}, \ldots, \lambda_{n}\right) \in \mathbb{R}^{n}$ and

$$
\mathcal{H}_{\lambda}:=\left\{A \in M_{n}(\mathbb{C}): A^{*}=A, \text { spectrum } A=\lambda\right\}
$$

be the $U(n)$-orbit of the matrix diagonal $\left(\lambda_{1}, \ldots, \lambda_{n}\right)$ in $\mathcal{H}$.

We identify $U(n)$-orbits in $\mathcal{H}$ with adjoint orbits in $\mathfrak{u}(n)$ by sending a matrix $A \in \mathcal{H}$ to the matrix $i A \in \mathfrak{u}(n)$. The pairing in $\mathfrak{u}(n)=i \mathcal{H}$ defined by

$$
(X, Y)=\operatorname{Trace}(X Y)
$$

allows us to identify $\mathfrak{u}(n)$ with $\mathfrak{u}(n)^{*}$, and adjoint orbits in $\mathfrak{u}(n)$ with coadjoint orbits in $\mathfrak{u}(n)^{*}$. So that, the $U(n)$-orbits $\mathcal{H}_{\lambda}$ in $\mathcal{H}$ can be identified with the coadjoit orbits in $\mathfrak{u}(n)^{*}$.

The coadjoint orbit associated with $\mathcal{H}_{\lambda}$ is regular when all the components of $\lambda \in \mathbb{R}^{n}$ are pairwise different, otherwise the coadjoint orbit is non-regular.

We identify $\mathcal{H}_{\lambda}$ with a coadjoint orbit in $\mathfrak{u}(n)^{*}$ and define on it a symplectic form $\omega_{\lambda}$ by pulling back the Kirillov-Kostant-Souriau form defined on the coadjoint orbit. We also endow $\mathcal{H}_{\lambda}$ with a complex structure $J_{\lambda}$, coming from the presentation of $\mathcal{H}_{\lambda}$ as a quotient of complex Lie groups $S l(n, \mathbb{C}) / P$, where $P \subset S l(n, \mathbb{C})$ is a parabolic subgroup of block upper triangular matrices. The triple $\left(\mathcal{H}_{\lambda}, \omega_{\lambda}, J_{\lambda}\right)$ is a Kähler manifold.

Let $\left\{e_{i}\right\}_{i=1}^{n}$ denote the standard basis of $\mathbb{R}^{n}$. Let $T=U(1)^{n} \subset U(n)$ be the standard maximal torus of $U(n)$ and $\mathfrak{t} \cong \mathbb{R}^{n}$ be its Lie algebra. We identify $\mathfrak{t}^{*}$ with $\mathfrak{t}$ via its standard inner product so that the standard basis $\left\{e_{i}\right\}_{i=1}^{n}$ of $\mathfrak{t} \cong \mathbb{R}^{n}$ is identified with the standard basis of projections of $\mathfrak{t}^{*}$, which is also the standard basis (as a $\mathbb{Z}$-module) of the weight lattice $\operatorname{Hom}\left(T, S^{1}\right) \subset \mathfrak{t}^{*}$. 
The restricted action of $T \subset U(n)$ on $\mathcal{H}_{\lambda}$ is Hamiltonian with momentum map

$$
\begin{aligned}
\mu: \mathcal{H}_{\lambda} & \rightarrow \mathfrak{t}^{*} \simeq \mathbb{R}^{n} \\
\left(a_{i j}\right) & \mapsto\left(a_{11}, \ldots, a_{n n}\right)
\end{aligned}
$$

The image of the momentum map is the convex hull of the momentum images of the fixed points of the action of $T$ on $\mathcal{H}_{\lambda}$, i.e., the image of $\mu$ is the convex hull of all possible permutations of the vector $\left(\lambda_{1}, \ldots, \lambda_{n}\right) \in \mathfrak{t}^{*} \cong \mathbb{R}^{n}$ (see, e.g., Audin [1, Chapter III], Guillemin [7]).

The $U(n)$-orbit $\mathcal{H}_{\lambda}$ together with the torus $T$ action is a GKM space, i.e., the closure of every connected component of the set

$$
\left\{x \in \mathcal{H}_{\lambda}: \operatorname{dim}_{\mathbb{C}}(T \cdot x)=1\right\}
$$

is a sphere (see e.g. Tymoczko [21], Guillemin, Holm and Zara [6]). The closure of $\left\{x \in \mathcal{H}_{\lambda}: \operatorname{dim}_{\mathbb{C}}(T \cdot x)=1\right\}$ is called the 1 -skeleton of $\mathcal{H}_{\lambda}$. The moment graph or GKM graph of $\mathcal{H}_{\lambda}$ is the image of its 1-skeleton under the momentum map. The vertices and edges of this graph are in correspondence with the $T$-fixed points of $\mathcal{H}_{\lambda}$ and the closures of the connected components of the 1 -skeleton of $\mathcal{H}_{\lambda}$, respectively.

Two $T$-fixed points $F, F^{\prime} \in \mathcal{H}_{\lambda}$ are connected by one connected component of the 1-skeleton of $\mathcal{H}_{\lambda}$ if and only if they differ by one transposition. We denote by $S_{F, F^{\prime}}^{2} \subset \mathcal{H}_{\lambda}$ the corresponding sphere associated to them.

We now want to compute the symplectic area of $S_{F, F^{\prime}}^{2} \subset \mathcal{H}_{\lambda}$ with respect to $\omega_{\lambda}$ in terms of $\lambda$. Let us suppose that $F$ and $F^{\prime}$ differ by the transposition $(i, j) \in S_{n}$ and the $(i, i)$-th component $F_{i} \in\left\{\lambda_{1}, \ldots, \lambda_{n}\right\}$ of $F$ is greater than its $(j, j)$-th component $F_{j} \in\left\{\lambda_{1}, \ldots, \lambda_{n}\right\}$. If $T^{\prime} \subset T$ is the codimension one torus that fixes $S_{F, F^{\prime}}^{2}$, there exists a torus of dimension one $S \subset T$ such that $T \cong T^{\prime} \times S$. We will use the identification $S:=\mathbb{R} / \mathbb{Z}$, which induces an isomorphism $\operatorname{Lie}(S) \cong \mathbb{R}$ leading to $\operatorname{Lie}(S)^{*} \cong \mathbb{R}$, mapping the lattice $\operatorname{Hom}\left(S, S^{1}\right) \subset \operatorname{Lie}(S)^{*}$ isomorphically to $\mathbb{Z} \subset \mathbb{R}$.

The action of $S$ on $S_{F, F^{\prime}}^{2}$ is Hamiltonian with momentum map

$$
\left.\iota^{*} \circ \mu\right|_{S_{F, F^{\prime}}^{2}}: S_{F, F^{\prime}}^{2} \rightarrow \operatorname{Lie}(S)^{*} \cong \mathbb{R}
$$

where $\iota: S \hookrightarrow T$ is the inclusion map. The momentum image of $S_{F, F^{\prime}}^{2}$ under $\left.\iota^{*} \circ \mu\right|_{S_{F, F^{\prime}}^{2}}$ is the segment line that joins $\iota^{*}(\mu(F))$ with $\iota^{*}\left(\mu\left(F^{\prime}\right)\right)$. Note that the weight of $T$ on $T_{F} S_{F, F^{\prime}}^{2}$ is equal to $e_{i}-e_{j}$, thus the weight of the action of $S$ on $T_{F} S_{F, F^{\prime}}^{2}$ is $\iota^{*}\left(e_{i}-e_{j}\right)$, an integer number. 
Let $\gamma:[0,1] \rightarrow S_{F, F^{\prime}}^{2} \hookrightarrow \mathcal{H}_{\lambda}$ be any smooth path from $F$ to $F^{\prime}$ and $c$ : $[0,1] \times S \rightarrow S_{F, F^{\prime}}^{2}$ be the map defined by $c(t, s):=s \cdot \gamma(t)$. Then,

$$
\int_{[0,1] \times S} c^{*}\left(\left.\omega_{\lambda}\right|_{S_{F, F^{\prime}}^{2}}\right)=\int_{0}^{1} \gamma^{*}\left(\iota_{\xi_{S_{F, F^{\prime}}}} \omega_{\lambda}\right)=\iota^{*}(\mu(F))-\iota^{*}\left(\mu\left(F^{\prime}\right)\right) .
$$

Note that the integral $\int_{[0,1] \times S} c^{*} \omega_{\lambda}$ is equal to the symplectic area of $S_{F, F^{\prime}}^{2}$ times the weight $\iota^{*}\left(e_{i}-e_{j}\right)$. Since $F-F^{\prime}=\left(F_{i}-F_{j}\right)\left(e_{i}-e_{j}\right)$, and $\iota^{*}(\mu(F))-$ $\iota^{*}\left(\mu\left(F^{\prime}\right)\right)=\left(F_{i}-F_{j}\right) \iota^{*}\left(e_{i}-e_{j}\right)$, we conclude that the symplectic area of $S_{F, F^{\prime}}^{2}$ is equal to $F_{i}-F_{j}$.

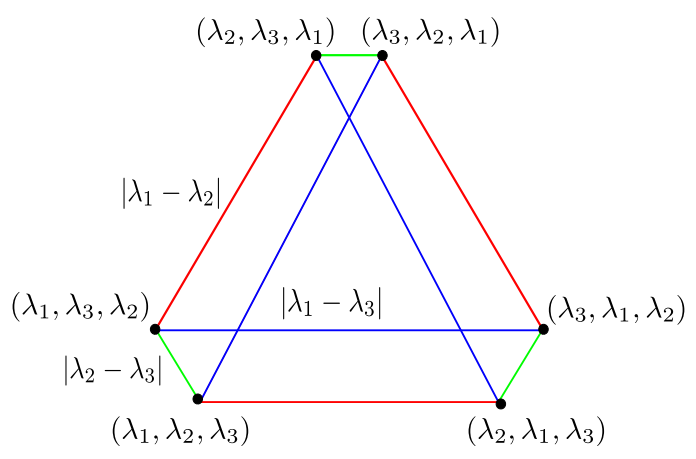

Figure 1: Moment graph of $\mathcal{H}_{\left(\lambda_{1}, \lambda_{2}, \lambda_{3}\right)}$ and some of its edges labeled with theirs corresponding symplectic areas.

Let $\lambda_{b_{1}}, \lambda_{b_{2}}, \ldots, \lambda_{b_{l}}$ be the pairwise different components of $\lambda \in \mathbb{R}^{n}$ with multiplicities $m_{1}, m_{2}, \ldots, m_{l}$, respectively. Let us assume without lost of generality that $\lambda$ is equal to

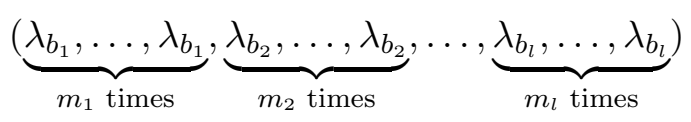

Let $a$ be the strictly increasing sequence of integers $0=a_{0}<a_{1}<a_{2}<$ $\cdots<a_{l}=n$ defined by $a_{j}=\sum_{i=1}^{j} m_{i}$ and let $F l(a ; n)$ be the set of increasing filtrations of $\mathbb{C}^{n}$ by complex subspaces

$$
0=V^{0} \subset V^{1} \subset V^{2} \subset \cdots \subset V^{l}=\mathbb{C}^{n}
$$

such that $\operatorname{dim}_{\mathbb{C}} V^{i}=a_{i}$.

Note that there is a naturally defined action of $\operatorname{Sl}(n, \mathbb{C})$ on $F l(a ; n)$. 
For a flag $V=\left(V^{1}, \ldots, V^{l}\right) \in F l(a ; n)$, denote by $P_{j}=P_{j}(V): \mathbb{C}^{n} \rightarrow \mathbb{C}^{n}$ the orthogonal projection onto $V_{j}$. We can form the Hermitian operator

$$
A_{\lambda}(V)=\sum_{j} \lambda_{b_{j}}\left(P_{j}-P_{j-1}\right) .
$$

The correspondence $V \mapsto A_{\lambda}(V)$ defines a diffeomorphism between $\mathrm{Fl}(a ; n)$ and $\mathcal{H}_{\lambda}$. This diffeomorphism defines by pullback a $U(n)$-invariant symplectic form on $F l(a ; n)$. It also defines an integrable almost complex structure on $\mathrm{Fl}(a ; n)$, and it allows us to define an action of $S l(n, \mathbb{C})$ on $\mathcal{H}_{\lambda}$ so the map $A_{\lambda}: F l(n, \mathbb{C}) \rightarrow \mathcal{H}_{\lambda}$ is a $\operatorname{Sl}(n, \mathbb{C})$-equivariant biholomorphism.

The homology of $F l(a, n)$ (and hence the homology of $\mathcal{H}_{\lambda}$ ) can be computed from the CW-structure of $F l(a ; n)$ coming from its Schubert cell decomposition.

Let $S_{n}$ be the group of permutations of $n$ elements. Recall that the length of a permutation is, by definition, equal to the smallest number of adjacent transpositions whose product is the permutation. Let $W_{a} \subset S_{n}$ be the subgroup generated by the simple transpositions $s_{i}=(i, i+1)$ for $i \notin$ $\left\{a_{1}, \ldots, a_{l}\right\}$. Let $W^{a} \subset S_{n}$ be the set of minimal length coset representatives of $S_{n} / W_{a}$. Let $F \in F l(a ; n)$ be the partial flag defined by

$$
F:=\mathbb{C}^{a_{1}} \subset \mathbb{C}^{a_{2}} \subset \cdots \subset \mathbb{C}^{a_{n}}=\mathbb{C}^{n}
$$

and $B$ be the standard Borel subgroup of $S l(n, \mathbb{C})$ of upper triangular matrices.

For a permutation $w \in W^{a}$, the Schubert cell $C_{w}$ is the orbit of the induced action of $B \subset S l(n, \mathbb{C})$ on $F l(a ; n)$ through $w \cdot F$. The Schubert variety $X_{w}$ is by definition the closure of the Schubert cell $C_{w}$.

For $w \in W^{a}$, the Schubert cell $C_{w}$ is isomorphic to an affine space of complex dimension equal to the length of $w$. The Schubert cells $\left\{C_{w}\right\}_{w \in W^{a}}$ define a $\mathrm{CW}$-complex for $\mathrm{Fl}(a ; n)$ with cells occurring only in even dimension. Thus, the fundamental classes $\left[X_{w}\right]$ of $X_{w}, w \in W^{a}$, are a free basis of $H_{*}(F l(a ; n), \mathbb{Z})$ as a $\mathbb{Z}$-module. Likewise, the Poincaré dual classes of $\left[X_{w}\right]$, $w \in W^{a}$, are a free basis of $H^{*}(F l(a ; n), \mathbb{Z})$ as a $\mathbb{Z}$-module.

The diffeomorphism $A_{\lambda}: F l(a ; n) \rightarrow \mathcal{H}_{\lambda}$ maps the Schubert cells $C_{w} \in$ $F l(a ; n), w \in W^{a}$, to the $B$-orbits of $w \cdot$ diagonal $\lambda$ in $\mathcal{H}_{\lambda}$. By abusing notation, we will denote the $B$-orbits of $w \cdot$ diagonal $\lambda$ in $\mathcal{H}_{\lambda}$ by $C_{w}$ and their closures by $X_{w}$ and refer to them as the Schubert cells and Schubert varieties associated to $w \in W^{a}$ in $\mathcal{H}_{\lambda}$, respectively. 
Remark 3.1. Note that $A_{\lambda}$ maps the Schubert varieties $X_{\left(a_{j}, a_{j}+1\right)} \subset$ $F l(a ; n)$ to the spheres $S_{\lambda,\left(a_{j}, a_{j}+1\right) \cdot \lambda}^{2} \subset \mathcal{H}_{\lambda}$. Thus, the fundamental classes of $S_{\lambda,\left(a_{j}, a_{j}+1\right) \cdot \lambda}^{2}, 1 \leq j \leq l$, conform a free Abelian basis of the homology group $H_{2}\left(\mathcal{H}_{\lambda}, \mathbb{Z}\right)$

\section{Upper bounds for the Gromov width of Grassmannian manifolds}

Karshon and Tolman [10] have found upper bounds for the Gromov width of Grassmannian manifolds by computing a Gromov-Witten invariant. In this section, we review this idea. This will allow us later to determine upper bounds for the Gromov width of flag manifolds by computing GromovWitten invariants on holomorphic fibrations whose fibers are isomorphic to Grassmannian manifolds

Now, we establish the convention that would be used during this section. Let $G(k, n)$ be the Grassmannian manifold of $k$-planes in $\mathbb{C}^{n}$. Let $\lambda \in \mathbb{R}^{n}$ with $k$ components equal to $\lambda_{1}$, and the other $n-k$ equal to $\lambda_{2}$, and $\mathcal{H}_{\lambda}=$ $\left\{A \in M_{n}(\mathbb{C}): A^{*}=A\right.$, spectrum $\left.A=\lambda\right\}$. The set of Hermitian matrices $\mathcal{H}_{\lambda}$ is diffeomorphic to the Grassmannian manifold $G(k, n)$.

Let $\left(\omega_{\lambda}, J_{\lambda}\right)$ be the Kähler structure of $\mathcal{H}_{\lambda} \cong G(k, n)$ defined in Section 3 . By Remark 3.1, the second homology group $H_{2}(G(k, n), \mathbb{Z})$ has one free abelian generator with positive symplectic area, let $A$ be this generator. Let $\mathcal{M}_{A}\left(G(k, n), J_{\lambda}\right)=\left\{u: \mathbb{C P}^{1} \rightarrow G(k, n): u\right.$ is $J_{\lambda^{-}}$holomorphic, $\left.u_{*}\left[\mathbb{C P}^{1}\right]=A\right\}$

be the moduli space of $J_{\lambda}$-holomorphic curves of degree $A$ defined on $G(k, n)$. We call any element of this moduli space a holomorphic line of the Grassmannian manifold $G(k, n)$.

For a holomorphic line $u: \mathbb{C P}^{1} \rightarrow G(k, n)$, we define the kernel of $u$ as the intersection of all the subspaces $V \subset \mathbb{C}^{n}$ that are in the image of $u$. Similarly, the span of $u$ is the linear span of these subspaces:

$$
\operatorname{ker}(u)=\bigcap_{V \in u\left(\mathbb{C P}^{1}\right)} V, \quad \operatorname{span}(u)=\sum_{V \in u\left(\mathbb{C P}^{1}\right)} V
$$

The kernel and span of $u$ are of dimension $k-1$ and $k+1$, respectively; and they determine the holomorphic line up to parametrization, i.e., if there is a holomorphic line $v: \mathbb{C P}^{1} \rightarrow G(k, n)$ such that $\operatorname{ker}(u)=\operatorname{ker}(v)$ and $\operatorname{span}(u)=$ $\operatorname{span}(v)$, then there exists $g: \mathbb{C P}^{1} \rightarrow \mathbb{C P}^{1} \in P S L(2 ; \mathbb{C})$ such that $v=u \circ g$. Moreover, $u\left(\mathbb{C P}^{1}\right)=\left\{V^{k} \in G(k, n): \operatorname{ker}(u) \subset V^{k} \subset \operatorname{span}(u)\right\} \subset G(k, n)$ (see 
e.g. Buch, Kresch and Tamvakis[3]). So

$$
\mathcal{M}_{A, 0}\left(G(k, n), J_{\lambda}\right):=\mathcal{M}_{A}\left(G(k, n), J_{\lambda}\right) / P S L(2, \mathbb{C}) \simeq F l(k-1, k+1 ; n)
$$

where $F l(k-1, k+1 ; n)$ denotes the partial flag manifold of complex subspaces sequences

$$
V^{k-1} \subset V^{k+1} \subset \mathbb{C}^{n}
$$

For $V=\left(V^{k-1}, V^{k+1}\right) \in F l(k-1, k+1 ; n)$, we will denote by $u_{V}$ the (unparameterized) holomorphic line

$$
\mathbb{C P}^{1} \simeq u_{V}=\left\{V^{k} \in G(k, n): V^{k-1} \subset V^{k} \subset V^{k+1}\right\} \subset G(k, n) .
$$

Let us consider the evaluation map

$$
\mathrm{ev}_{J_{\lambda}}^{2}: \mathcal{M}_{A}\left(G(k, n), J_{\lambda}\right) \times_{P S L(2, \mathbb{C})}\left(\mathbb{C P}^{1}\right)^{2} \rightarrow G(k, n)^{2}
$$

We want to find a compact complex submanifold $X \subset G(k, n)$ such that for a generic point $p$ in $G(k, n)$ the evaluation map $\mathrm{ev}_{J_{\lambda}}^{2}$ would be transverse to $(\{p\} \times X) \subset G(k, n)^{2}, \operatorname{dim}_{\mathbb{C}}\left(\mathcal{M}_{A}\left(G(k, n), J_{\lambda}\right) \times_{P S L(2, \mathbb{C})}\left(\mathbb{C P}^{1}\right)^{2}\right)+$ $\operatorname{dim}_{\mathbb{C}} X$ would be equal to $2 \operatorname{dim}_{\mathbb{C}} G(k, n)$, and the number of holomorphic lines in $\mathcal{M}_{A, 0}\left(G(k, n), J_{\lambda}\right)$ that pass through $p$ and $X$ would be different from zero. If so, the Gromov-Witten invariant $\mathrm{GW}_{A, 2}^{J_{\lambda}}(\mathrm{PD}[p], \mathrm{PD}[X])$ would be different from zero and by Theorem 2.2 and Remark 2.3 , we will have that

$$
\operatorname{Gwidth}\left(\mathcal{H}_{\lambda}, \omega_{\lambda}\right) \leq \omega_{\lambda}(A)=\left|\lambda_{1}-\lambda_{2}\right|
$$

We claim that $X=\left\{V^{k} \in G(k, n): \mathbb{C} \subset V^{k} \subset \mathbb{C}^{n-1}\right\} \cong G(k-1, n-2)$ $\subset G(k, n)$ satisfies all these conditions.

Proving that the evaluation map $\mathrm{ev}_{J_{\lambda}}^{2}$ is transverse to $(\{p\} \times X) \subset$ $G(k, n)^{2}$ can be obtained as a consequence of the Bertini-Kleiman Transversality Theorem:

Theorem 4.1. Let $f: U \rightarrow V$ be a smooth map between smooth manifolds and let $G$ be a Lie group that acts transitively on $V$. Let $Z$ be an arbitrary submanifold of $V$ and $G^{\text {reg }}$ be the set of elements $g \in G$ for which $f$ is transverse to $g Z$. Then, $G^{r e g}$ is a set of the second category in $G$.

Proof. See proof of Proposition 7.4.5 in McDuff and Salamon [16]

We now prove that indeed the Gromov-Witten invariant $\mathrm{GW}_{A, 2}^{J_{\lambda}}(\mathrm{PD}[p]$, $\mathrm{PD}[X])$ is different from zero. 
Lemma 4.2. Let $X=\left\{V^{k} \in G(k, n): \mathbb{C} \subset V^{k} \subset \mathbb{C}^{n-1}\right\} \simeq G(k-1, n-2)$ and $p \in G(k, n)$. Then

$$
\mathrm{GW}_{A, 2}^{J_{\lambda}}(\mathrm{PD}[p], \mathrm{PD}[X])=1
$$

Proof. The complex dimension of $X$ is equal to $(n-k-1)(k-1)$ and as a consequence $X$ satisfies the dimensional constraint:

$$
\begin{aligned}
& \operatorname{dim}_{\mathbb{C}}\left(\mathcal{M}_{A, 2}\left(G(k, n), J_{\lambda}\right)\right)+\operatorname{dim}_{\mathbb{C}} X \\
= & \operatorname{dim}_{\mathbb{C}} F l(k-1, k+1 ; n)+2+\operatorname{dim}_{\mathbb{C}} X \\
= & \frac{n^{2}-(k-1)^{2}-2^{2}-(n-k-1)^{2}}{2}+2+(n-k-1)(k-1) \\
= & 2 \operatorname{dim}_{\mathbb{C}} G(k, n) .
\end{aligned}
$$

Assume now that $p=W^{k}$ is a $k$-dimensional subspace of $\mathbb{C}^{n}$ that does not contain $\mathbb{C}$ and transversally intersects $\mathbb{C}^{n-1}$. We claim that $\left(\operatorname{ev}_{J_{\lambda}}^{2}\right)^{-1}(\{p\}$ $\times X)$ consists of just one element, i.e., there is a unique (unparameterized) holomorphic line in $G(k, n)$ that intersects $X$ and passes through $W^{k}$.

Let $V=\left(V^{k-1}, V^{k+1}\right) \in F l(k-1, k+1 ; n)$ be such that the holomorphic line $u_{V}$ passes through both $X$ and $p$. So there exists $V^{k} \in X$ (that is, $\mathbb{C} \subset V^{k} \subset \mathbb{C}^{n-1}$ ) and $V^{k-1} \subset V^{k} \subset V^{k+1}$. Moreover we have $V^{k-1} \subset W^{k} \subset$ $V^{k+1}\left(W^{k}\right.$ is $\left.p\right)$.

Note that, we have inclusions $V^{k-1} \subset \mathbb{C}^{n-1}$ and $V^{k-1} \subset W^{k}$. Thus $V^{k-1}$ $\subset W^{k} \cap \mathbb{C}^{n-1}$. But $W^{k} \cap \mathbb{C}^{n-1}$ is a $(k-1)$-dimensional vector subspace because the intersection is transverse. Thus $V^{k-1}=W^{k} \cap \mathbb{C}^{n-1}$. The intersection $V^{k-1}=W^{k} \cap \mathbb{C}^{n-1}$ does not contain $\mathbb{C}$. So there exists a unique $k$ dimensional vector space $U^{k}$ such that $V^{k-1} \subset U^{k}$ and $\mathbb{C} \subset U^{k} \subset \mathbb{C}^{n-1}$. This vector space is $U^{k}=V^{k-1} \oplus \mathbb{C}$. Thus, $V^{k}=V^{k-1} \oplus \mathbb{C}$. The vector space $V^{k+1}$ contains $W^{k}$ and $V^{k}=V^{k-1} \oplus \mathbb{C}$. Observe that $V^{k}$ is different from $W^{k}$ because $V^{k}$ contains $\mathbb{C}$ and $W^{k}$ does not. Therefore $V^{k+1}=W^{k}+V^{k}$.

In conclusion $\left(V^{k-1}, V^{k+1}\right)=\left(W^{k} \cap \mathbb{C}^{n-1}, W^{k}+\left(\left(W^{k} \cap \mathbb{C}^{n-1}\right) \oplus \mathbb{C}\right)\right)$, which determines a unique holomorphic line that intersects $X$ and passes through $W^{k}$. The figure shown below illustrates graphically the idea of this argument.

Note that if $p=W^{k}$ is a $k$-dimensional subspace of $\mathbb{C}^{n}$ that either contains $\mathbb{C}$ or is contained in $\mathbb{C}^{n-1}$, then $\left(\mathrm{ev}_{J_{\lambda}}^{2}\right)^{-1}(\{p\} \times X)$ consists of an infinite number of elements.

We now prove that the evaluation map

$$
\mathrm{ev}_{J_{\lambda}}^{2}: \mathcal{M}_{A, 2}\left(J_{\lambda}\right) \rightarrow G(k, n)^{2}
$$




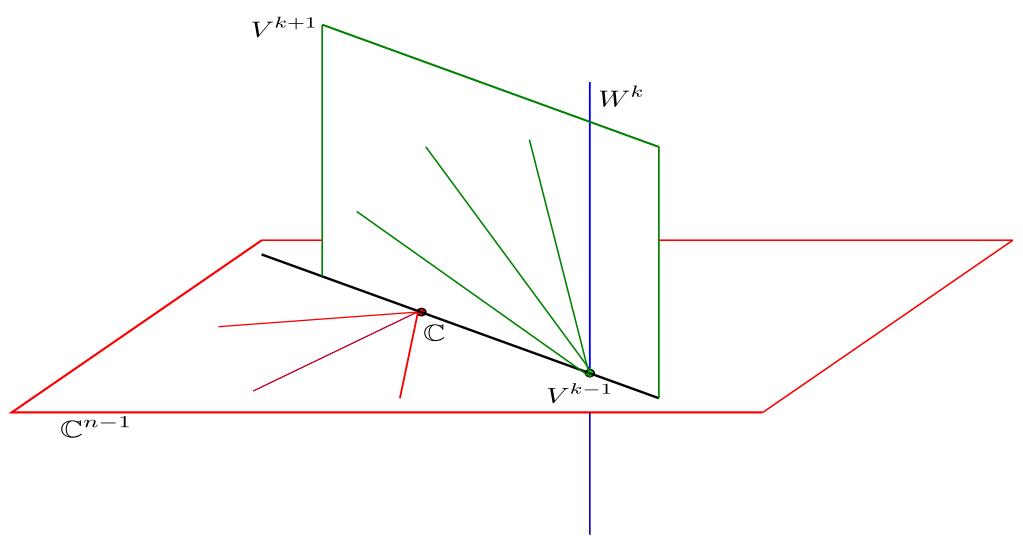

Figure 2: The vector spaces between $V^{k-1}$ and $V^{k+1}$ (green) corresponds to the unique holomorphic line in the Grassmannian manifold $G(k, n)$ passing through the generic point $W^{k}$ (blue) and $X$ (red). The intersection of the holomorphic line with $X$ is the $k$-dimensional vector spanned by $V^{k-1}$ and $\mathbb{C}$ (black).

is transverse to $(\{p\} \times X) \subset G(k, n)^{2}$. The group $S l(n, \mathbb{C})$ acts transitively and holomorphically on $G(k, n)$ so as a consequence there exists $h \in$ $S l(n, \mathbb{C})$ such that $\operatorname{ev}_{J_{\lambda}}^{2} \pitchfork(\{h \cdot p\} \times X) \subset G(k, n)^{2}$ and thus the preimage $\left(\mathrm{ev}_{J_{\lambda}}^{2}\right)^{-1}(\{h \cdot p\} \times X)$ consists of just one point (the number of elements of the preimage $\left(\mathrm{ev}_{J_{\lambda}}^{2}\right)^{-1}(\{h \cdot p\} \times X)$ is either one or infinite, but if the evaluation map is transverse to $\{h \cdot p\} \times X$ it has to be necessarily one), by Proposition 7.4.5 of Mcduff and Salamon [16] the Gromov-Witten invariant $\mathrm{GW}_{A, 2}^{J_{\lambda}}(\mathrm{PD}[p], \mathrm{PD}[X])$ is positive, so in conclusion

$$
\mathrm{GW}_{A, 2}^{J_{\lambda}}(\mathrm{PD}[p], \mathrm{PD}[X])=\mathrm{GW}_{A, 2}^{J_{\lambda}}(\mathrm{PD}[h \cdot p], \mathrm{PD}[X])=1
$$

We have proved that for Grassmannian manifolds there is a non-vanishing Gromov-Witten invariant with one of its constrains being Poincaré dual to the class of a point. This would imply that the Gromov width of a Grassmannian manifold is bounded from above by the symplectic area of any line of the Grassmannian manifold. In summary, we have the following result:

Theorem 4.3 (Karshon-Tolman, G. Lu). Let $\lambda \in \mathbb{R}^{n}$ with $k$ components equal to $\lambda_{1}$, and the other $n-k$ equal to $\lambda_{2}$. Let

$$
\mathcal{H}_{\lambda}=\left\{A \in M_{n}(\mathbb{C}): A^{*}=A, \text { spectrum } A=\lambda\right\}
$$


and let $\omega_{\lambda}$ be the Kirillov-Kostant-Souriau form defined on $\mathcal{H}_{\lambda}$. Then,

$$
\operatorname{Gwidth}\left(\mathcal{H}_{\lambda}, \omega_{\lambda}\right) \leq\left|\lambda_{1}-\lambda_{2}\right|
$$

Proof. First notice that the fundamental class $A$ of any line in $G(k, n)$ is $\omega_{\lambda^{-}}$ indecomposable. This is because the homology group $H_{2}(G(k, n), \mathbb{Z})$ is cyclic generated by this fundamental class. Thus, the Gromov-Witten invariant $\mathrm{GW}_{A, 2}$ is well defined and independent of the almost complex structure. The result now follows from Remark 2.3 and Lemma 4.2 , and the fact that the symplectic area of $A$ with respect to $\omega_{\lambda}$ is equal to $\left|\lambda_{1}-\lambda_{2}\right|$.

\section{Upper bounds for the Gromov width of flag manifolds}

In his Ph.D thesis Zoghi [22] has considered the problem of determining the Gromov width of regular coadjoint orbits of compact Lie groups. We start this section by first stating Zoghi's results, and then we show how to extend his results to coadjoint orbits that may not be necessarily regular.

Let $\lambda=\left(\lambda_{1}, \ldots, \lambda_{n}\right) \in \mathbb{R}^{n}, \mathcal{H}_{\lambda}=\left\{A \in M_{n}(\mathbb{C}): A^{*}=A, \operatorname{spectrum} A=\lambda\right\}$ and $\left(\omega_{\lambda}, J_{\lambda}\right)$ be the Kähler structure of $\mathcal{H}_{\lambda}$ defined in Section 3 . The following Theorem appears in Zoghi's Ph.D thesis [22] as one of its main results:

Theorem 5.1 (Zoghi). Let us assume that all the components of $\lambda \in \mathbb{R}^{n}$ are pairwise different and suppose that there are integers $1 \leq i, j \leq n$ such that any difference of eigenvalues $\lambda_{i^{\prime}}-\lambda_{j^{\prime}}$ is an integer multiple of $\lambda_{i}-\lambda_{j}$, then

$$
\operatorname{Gwidth}\left(\mathcal{H}_{\lambda}, \omega_{\lambda}\right) \leq\left|\lambda_{i}-\lambda_{j}\right|
$$

Now we show how to extend Zoghi's result to sets of coadjoint orbits that are not necessarily regular. But first we state the following lemma:

Lemma 5.2. Let $B \subset S l(n, \mathbb{C})$ be the Borel subgroup of upper triangular matrices and $P \subset S l(n, \mathbb{C})$ be any parabolic subgroup of block upper triangular matrices. Let $X$ be an algebraic $B$-variety and $\pi: X \rightarrow S l(n, \mathbb{C}) / P$ be a B-equivariant map. If $\Omega$ is the B-stable open dense Schubert cell of $\operatorname{Sl}(n, \mathbb{C}) / P$, then $\pi$ is a trivial fibration over $\Omega$.

Proof. Let $x_{0} \in \Omega$ be any point and $U \subset B$ be the unipotent radical of $P$. The map $s: U \rightarrow \Omega$ defined by $g \mapsto g \cdot x_{0}$ is an isomorphism. Let $t: \Omega \rightarrow U$ 
be the inverse function of $s$. The map

$$
\begin{aligned}
\psi: \Omega \times \pi^{-1}\left(x_{0}\right) & \rightarrow \pi^{-1}(\Omega) \\
(x, y) & \mapsto t(x) \cdot y
\end{aligned}
$$

is an isomorphism with inverse given by

$$
\begin{aligned}
\psi^{-1}: \pi^{-1}(\Omega) & \rightarrow \Omega \times \pi^{-1}\left(x_{0}\right) \\
m & \mapsto\left(\pi(m), t(\pi(m))^{-1} \cdot m\right) .
\end{aligned}
$$

Theorem 5.3. Let $\lambda=\left(\lambda_{1}, \ldots, \lambda_{n}\right) \in \mathbb{R}^{n}$. Suppose that there are integers $1 \leq i, j \leq n$ such that any difference of eigenvalues $\lambda_{i^{\prime}}-\lambda_{j^{\prime}}$ is an integer multiple of $\lambda_{i}-\lambda_{j}$, then

$$
\operatorname{Gwidth}\left(\mathcal{H}_{\lambda}, \omega_{\lambda}\right) \leq\left|\lambda_{i}-\lambda_{j}\right|
$$

Proof. The idea of the proof is, as before, to prove that a certain GromovWitten invariant, with one of its constraints being Poincaré dual to the fundamental class of a point, is different from zero.

Let $\lambda_{b_{1}}, \lambda_{b_{2}}, \ldots, \lambda_{b_{l}}$ be the pairwise different components of $\lambda \in \mathbb{R}^{n}$ with multiplicities $m_{1}, m_{2}, \ldots, m_{l}$, respectively. Let us assume without lost of generality that $\lambda$ is equal to

$$
(\underbrace{\lambda_{b_{1}}, \ldots, \lambda_{b_{1}}}_{m_{1} \text { times }}, \underbrace{\lambda_{b_{2}}, \ldots, \lambda_{b_{2}}}_{m_{2} \text { times }}, \ldots, \underbrace{\lambda_{b_{l}}, \ldots, \lambda_{b_{l}}}_{m_{l} \text { times }})
$$

After reordering the components of $\lambda$ if necessary, we will assume that any difference of the form $\lambda_{i^{\prime}}-\lambda_{j^{\prime}}$ is an integer multiple of $\lambda_{b_{2}}-\lambda_{b_{1}}$.

We know that $\mathcal{H}_{\lambda} \simeq F l(a ; n)$, where $a$ is the strictly increasing sequence of integers $0=a_{0}<a_{1}<\cdots<a_{l}=n$ defined by $a_{k}=\sum_{r=1}^{k} m_{r}$, for $1 \leq$ $k \leq l$.

We will endow $F l(a ; n)$ with a Kähler structure coming from its identification with $\mathcal{H}_{\lambda}$. This Kähler structure and the one defined on $\mathcal{H}_{\lambda}$ would be denoted indistinguishably by $\left(\omega_{\lambda}, J_{\lambda}\right)$.

Let $a^{\prime}$ be the sequence of integer numbers $a_{2}<\cdots<a_{l}=n$, and $\operatorname{Fl}\left(a^{\prime} ; n\right)$ be the corresponding partial flag manifold. Let $W_{a} \subset S_{n}$ be the subgroup generated by the simple transpositions $s_{i}=(i, i+1)$ for $i \notin\left\{a_{1}, \ldots, a_{l}\right\}$. Let $W^{a} \subset S_{n}$ be minimal length coset representatives of $S_{n} / W_{a}$. Likewise, we define $W_{a^{\prime}}$ and $W^{a^{\prime}}$. Schubert varieties of $F l(a ; n)$ and $F l\left(a^{\prime} ; n\right)$ are 
parametrized by $W^{a}$ and $W^{a^{\prime}}$, respectively. To avoid confusions, we will denote the Schubert varieties in $F l(a ; n)$ by $X_{\bullet}$ and the Schubert varieties in $F l\left(a^{\prime} ; n\right)$ by $X_{\bullet}^{\prime}$. A similar thing will be done with the Schubert cells.

For the permutation $\left(a_{1}, a_{1}+1\right) \in W^{a}$, let $X_{\left(a_{1}, a_{1}+1\right)}$ be the standard Schubert variety in $F l(a ; n)$ associated to it and let $A$ be the the fundamental class of this Schubert variety. Note that, by assumption, the symplectic area $\omega_{\lambda}(A)=\left|\lambda_{b_{2}}-\lambda_{b_{1}}\right|$ is a generator of the cyclic image $\omega_{\lambda}\left(H_{2}(F l(a ; n), \mathbb{Z})\right)$, which implies that $A$ is a $\omega_{\lambda}$-indecomposable homology class. As a consequence, the Gromov-Witten invariant $\mathrm{GW}_{A, k}$ is well defined.

We have a holomorphic projection

$$
\begin{aligned}
\pi: F l(a ; n) & \rightarrow F l\left(a^{\prime} ; n\right) \\
V^{a_{1}} \subset V^{a_{2}} \subset \cdots \subset V^{a_{l}}=\mathbb{C}^{n} & \mapsto V^{a_{2}} \subset \cdots \subset V^{a_{l}}=\mathbb{C}^{n}
\end{aligned}
$$

whose fibers are isomorphic to the Grassmanian manifold $G\left(a_{1}, a_{2}\right)$.

The set of minimal length representatives $W_{a^{\prime}}^{a}$ of $W_{a^{\prime}}$ on $W_{a}$ parameterizes Schubert varieties on a fiber of $\pi$. Note that $\left(a_{1}, a_{1}+1\right) \in W_{a^{\prime}}^{a}$, so in particular $\pi_{*}(A)=0$.

Let $\tilde{w}$ be the permutation in $W_{a^{\prime}}^{a}$ that represents in a fiber a Grassmannian manifold isomorphic to $G\left(a_{1}-1, a_{2}-2\right)$. Let $w^{\prime}$ be the longest element in $W^{a^{\prime}}$. The Schubert cell $C_{w^{\prime}}^{\prime}$ is open and dense in $F l\left(a^{\prime} ; n\right)$. By the previous Lemma, the restriction map

$$
\left.\pi\right|_{X_{w^{\prime} \tilde{w}}}: X_{w^{\prime} \tilde{w}} \rightarrow \operatorname{Fl}\left(a^{\prime} ; n\right)
$$

is a trivial fibration over $C_{w^{\prime}}^{\prime}$ with fiber isomorphic to $G\left(a_{1}-1, a_{2}-2\right)$.

Now we want to count the number of holomorphic curves of degree $A$ passing through a generic point $p \in F l(a ; n)$ and $X_{w^{\prime}} \tilde{w} \subset F l(a ; n)$. Let $u$ : $\mathbb{C P}^{1} \rightarrow F l(a ; n)$ be one of such holomorphic curves. The composition $\pi \circ u$ is holomorphic and $(\pi \circ u)_{*}\left[\mathbb{C P}^{1}\right]=\pi_{*}(A)=0$. Since $F l\left(a^{\prime} ; n\right)$ is a compact and connected Kähler manifold, the map $\pi \circ u$ is constant, which means that the image of $u: \mathbb{C P}^{1} \rightarrow F l(a ; n)$ lies entirely in the fiber $\pi^{-1}(p) \cong G\left(a_{1}, a_{2}\right)$ of $\pi$ : $F l(a ; n) \rightarrow F l\left(a^{\prime} ; n\right)$. Moreover, $u: \mathbb{C P}^{1} \rightarrow \pi^{-1}(p) \cong G\left(a_{1}, a_{2}\right) \subset F l(a ; n)$ is a holomorphic line of the fiber $\pi^{-1}(p) \cong G\left(a_{1}, a_{2}\right)$. If $\pi(p) \in C_{w^{\prime}}$, then the fiber $\pi^{-1}(p)$ intersects $X_{w^{\prime} \tilde{w}}$ in a variety isomorphic to $G\left(a_{1}-1, a_{2}-2\right)$. Since there is just one holomorphic line passing through a generic point and $G\left(a_{1}-1, a_{2}-2\right)$ in $G\left(a_{1}, a_{2}\right)$ (by Lemma 4.2 , we conclude that

$$
\mathrm{GW}_{A, 2}^{J_{\lambda}}\left(\operatorname{PD}[p], \operatorname{PD}\left[X_{w^{\prime}} \tilde{w}\right]\right)=1
$$


Thus, by Theorem 2.2 and Remark 2.3 .

$$
\operatorname{Gwidth}\left(\mathcal{H}_{\lambda}, \omega_{\lambda}\right) \leq \omega_{\lambda}(A)=\left|\lambda_{b_{2}}-\lambda_{b_{1}}\right|
$$

\section{Acknowledgments}

I would like to thank Yael Karshon for letting me know about this problem and for encouraging me during the writing process of this paper. I also would like to thank Milena Pabiniak for useful conversations and helpful comments on the first draft.

This research is partially supported by the Natural Sciences and Engineering Research Council of Canada.

\section{References}

[1] Michèle Audin, Torus actions on symplectic manifolds. Progress in Mathematics, volume 93, Birkhäuser Verlag, Basel, revised edition, 2004.

[2] Paul Biran, From symplectic packing to algebraic geometry and back. In: European Congress of Mathematics, Vol. II (Barcelona, 2000), Progr. Math., volume 202, pages 507-524. Birkhäuser, Basel, 2001.

[3] Anders Skovsted Buch, Andrew Kresch and Harry Tamvakis, GromovWitten invariants on Grassmannians. J. Amer. Math. Soc., 16(4):901915 (electronic), 2003.

[4] Kenji Fukaya and Kaoru Ono, Arnold conjecture and Gromov-Witten invariant for general symplectic manifolds. In: The Arnoldfest (Toronto, ON, 1997), Fields Inst. Commun., volume 24, pages 173-190. Amer. Math. Soc., Providence, RI, 1999.

[5] M. Gromov, Pseudoholomorphic curves in symplectic manifolds. Invent. Math., 82(2):307-347, 1985.

[6] V. Guillemin, T. Holm and C. Zara, A GKM description of the equivariant cohomology ring of a homogeneous space. J. Algebraic Combin., $\mathbf{2 3}(1): 21-41,2006$.

[7] Victor Guillemin, Moment maps and combinatorial invariants of Hamiltonian $T^{n}$-spaces. Progress in Mathematics, volume 122, Birkhäuser Boston Inc., Boston, MA, 1994. 
[8] Helmut Hofer, Krzysztof Wysocki and Eduard Zehnder, Integration theory on the zero sets of polyfold Fredholm sections. Mathematische Annalen 346 (2010), 139-198.

[9] Helmut Hofer, Krzysztof Wysocki and Eduard Zehnder, A general Fredholm theory. II. Implicit function theorems. Geom. Funct. Anal., 19(1):206-293, 2009.

[10] Yael Karshon and Susan Tolman, The Gromov width of complex Grassmannians. Algebr. Geom. Topol., 5:911-922 (electronic), 2005.

[11] A. A. Kirillov, Lectures on the orbit method. Graduate Studies in Mathematics, volume 64, American Mathematical Society, Providence, RI, 2004.

[12] Jun Li and Gang Tian, Virtual moduli cycles and Gromov-Witten invariants of general symplectic manifolds. In: Topics in symplectic 4manifolds (Irvine, CA, 1996), First Int. Press Lect. Ser., I, pages 47-83, Int. Press, Cambridge, MA, 1998.

[13] Guangcun Lu, Gromov-Witten invariants and pseudo symplectic capacities. Israel J. Math., 156:1-63, 2006.

[14] Guangcun Lu, Symplectic capacities of toric manifolds and related results. Nagoya Math. J., 181:149-184, 2006.

[15] Dusa McDuff and Leonid Polterovich, Symplectic packings and algebraic geometry. Invent. Math., 115(3):405-434, 1994. With an appendix by Yael Karshon.

[16] Dusa McDuff and Dietmar Salamon, J-holomorphic curves and symplectic topology. American Mathematical Society Colloquium Publications, volume 52, American Mathematical Society, Providence, RI, 2004.

[17] Milena Pabiniak, Lower bounds for Gromov width of coadjoint orbits in $U(n)$. arXiv:1109.0943v1, 2011.

[18] Milena Pabiniak, Gromov width of non-regular coadjoint orbits of $U(n)$, $S O(2 n)$ and $S O(2 n+1)$. Mathematical Research Letters, 21 (2014), 187-205.

[19] Yongbin Ruan, Virtual neighborhoods and pseudo-holomorphic curves. In: Proceedings of 6th Gökova Geometry-Topology Conference, volume 23, pages 161-231, 1999. 
[20] Bernd Siebert, Symplectic Gromov-Witten invariants. New trends in algebraic geometry (Warwick, 1996), London Math. Soc. Lecture Note Ser., 264 (1999), 375-424.

[21] Julianna S. Tymoczko, An introduction to equivariant cohomology and homology, following Goresky, Kottwitz, and MacPherson. In: nowbird lectures in algebraic geometry, Contemp. Math., volume 388, pages 169188, Amer. Math. Soc., Providence, RI, 2005.

[22] Masrour Zoghi, The Gromov Width of Coadjoint Orbits of Compact Lie Groups. Page 91, 2010. Thesis (Ph.D.)-University of Toronto (Canada).

Department of Mathematics, University of Toronto

Toronto, ON M5S 2E4, CANADA

E-mail address: alexander.caviedescastro@mail.utoronto.ca

Received February 26, 2013 